\title{
Articulación de la proyección social con la gestión universitaria en innovación social. Una mirada hacia la realidad del entorno y la contribución hacia él
}

\section{Irina Margarita Jurado Paz}

Corporación Universitaria Autónoma de Nariño, Pasto, Colombia, irina.jurado@aunar.edu.co

\begin{abstract}
Resumen
A partir de la experiencia vivida en el Programa de Internado Rural Interuniversitario -PIRI-, ejercicio académico interinstitucional $y$ extracurricular que ha permitido fortalecer la gestión de emprendimiento e innovación social al interior de la Corporación Universitaria Autónoma de Nariño; nace la necesidad de hacer un análisis sobre la innovación social, y cómo ésta contribuye a resolver problemas que afectan a una comunidad en particular, centrándose en las comunidades conectado a las personas con el objetivo de crear valor, así como, en conjunto visibilizando el conocimiento las oportunidades y alternativas desde una perspectiva interdisciplinaria $y$ abierta.
\end{abstract}

En Latinoamérica se ha empezado a realizar a través de un contexto académico, en especial por parte de las universidades, desde los ejercicios de proyección social, para vincular a los estudiantes con el entorno y la sociedad, a partir de los cuales es posible sintetizar lecciones aprendidas de cara a la configuración de modelos de gestión integral de los dos conceptos como herramientas pedagógicas para el fortalecimiento de las competencias propias de un profesional del siglo XXI. Con base en la estructura generada dentro de esta práctica académica, se permite que los futuros profesionales fortalezcan sus competencias ciudadanas, básicas y potencializar las técnicas o especificas a partir de un trabajo participativo.

Palabras clave: Innovación social, proyección social universitaria, gestión universitaria y formación profesional integral. 
Articulación de la proyección social con la gestión universitaria en innovación social. Una mirada hacia la realidad del entorno y la contribución hacia él

\section{Introducción}

Las condiciones sociales actuales se caracterizan por tener un mundo cada vez menos dividido, es decir, las fronteras son menos visibles en la actualidad, sin embargo, las desigualdades y los rezagos siguen siendo unas condiciones que fortalece la pobreza, agudiza la violencia y afecta las condiciones de vida de sus habitantes. Estas problemáticas requieren de intervenciones diferentes, con propuestas alternativas, que incluso provengan de quienes están siendo afectados por los flagelos y no por "ángeles de la guarda" extranjeros.

La innovación social, de acuerdo con la definición establecida por la Comisión Europea "consiste en encontrar nuevas formas de satisfacer las necesidades sociales, que no están adecuadamente cubiertas por el mercado o el sector público... o en producir los cambios de comportamiento necesarios para resolver los grandes retos de la sociedad... capacitando a la ciudadanía y generando nuevas relaciones sociales y nuevos modelos de colaboración. Son, por tanto, al mismo tiempo innovadoras en sí mismas y útiles para capacitar a la sociedad a innovar...”. (EUROPEAN COMMISSION, 2010)

$\mathrm{Al}$ analizar este concepto y articularlo con la labor que debe hacer la universidad en temas sociales, se identifica importantes puntos de intersección, puesto que las instituciones de educación superior tienen como uno de sus factores misionales la proyección hacia la comunidad con un sentido social especialmente, y desde allí podría apoyarse en lo que se entiende como innovación social para generar una real contribución a la construcción de soluciones a las problemáticas que está afrontando la sociedad.

En la Corporación Universitaria Autónoma de Nariño -AUNAR-, institución de educación superior con sede principal en la ciudad de Pasto, sur de Colombia, se ha trabajado desde hace tres años y manera significativa con el Programa de Internado Rural Interdisciplinario, ejercicio académico que fue iniciado por la Universidad de la Frontera en Chile hace más de 25 años y que se fundamenta en la aplicación de la investigación acción participativa con la comunidad.

Esta experiencia académica, permite llevar a través de ejercicios prácticos la innovación social hacia un contexto del desarrollo comunitario y social de territorios enmarcados por un sinnúmero de problemáticas que están afectando a sus pobladores. Dentro de este programa, los jóvenes universitarios desarrollan competencias a partir de su posibilidad de trabajar con la comunidad, haciendo con los pobladores importantes equipos sinérgicos en donde los conocimientos técnicos de unos, se complementan, con las experiencias de vida y 
las habilidades de los otros, y en conjunto potencializan las oportunidades de identificar problemas, definir soluciones, diseñarlas e implementarlas.

Al interior de este documento, el lector podrá encontrar cómo éstas prácticas académicas son un excelente instrumento para permitir que las instituciones de educación superior cumplan de la mejor manera posible, una de sus funciones misionales, como lo es la proyección social o hacia la comunidad, en donde es posible, apoyarse en la innovación social para alcanzar una contribución más significativa en la construcción de soluciones oportunas a los problema que afronta la sociedad, y a su vez, aprovecha estos espacios de trabajo con el entorno para fortalecer las competencias profesionales de los jóvenes universitarios, así como también, el desarrollo y crecimiento humano tanto del equipo docente, directivo y administrativo, es decir, es una relación de múltiples beneficios para diferentes actores que intervienen en el proceso.

Finalmente, este ejercicio resultado de un proceso investigativo, desea contribuir al debate académico de cómo las universidades deben interactuar con su entorno, no solo para entregarle a la sociedad un talento humano fortalecido sino también para desarrollar estrategias en donde la relación Universidad-sociedad sea mucho más provechosa para todos los involucrados.

\section{Marco teórico}

Para hacer una referenciación teórica, conceptual y contextual que facilite la comprensión por parte del lector sobre el desarrollo del proceso investigativo objeto del presente documento, se expone a continuación, un resumen sobre los desafíos de la Universidad en el siglo XXI, su relación con la innovación social, la metodología de Investigación Acción Participativa-IAP- y su relación con el contexto de la Corporación Universitaria Autónoma de Nariño y el Programa de Inserción Rural Interdisciplinaria -PIRI-.

\section{A. Función de la Universidad en el siglo XXI:}

Sin lugar a dudas, la sociedad actual está enmarcada por vivir la era del conocimiento, el desarrollo tecnológico y la globalización, situaciones que traen consigo una característica principal en casi todos los ambientes, el cambio continuo y permanente, a tal punto, que aquello considerado hoy como novedoso, en menos de lo imaginado, pasa a ser percibido como desactualizado o fuera de contexto.

Esta condición también ha afectado a las universidades, en especial, porque en pleno siglo XXI han enfrentado transformaciones muy significativas para estar acorde a las necesidades y desafíos actuales, más aún, cuando en el momento, se considera como principal función de estas organizaciones la generación de pensamientos cuya evolución es la sabiduría para 
ser, no solo entendida por la mente humana, sino para implementarse enmarco de los entornos altamente cambiantes y competitivos.

Dentro de este panorama actual de la sociedad, una de las labores que deberán cumplir las universidades es la de abordar la formación profesional del talento humano con unas competencias integrales, general y humanistas; porque de nada sirve que se genere unos saberes técnicos y aislados, puesto que estas habilidades requerirán de otras condiciones u otros conocimientos par realmente ser útiles; en otras palabras, las universidades deberán renunciar a la "supe formación específica" y pasar a una formación humanista, integral y crítica.

Las condiciones antes descritas, obliga a las Instituciones de Educación Superior a generar estrategias innovadoras en donde puedan conjugar el desarrollo de conocimientos técnicos prácticos y aplicados, y al mismo tiempo, fortalece la generación de competencias humanas, sociales y ciudadanas que le permitan al nuevo profesional ser competitivo, pertinente e idóneo a las condiciones propias de su entorno.

Las preguntas que surgen ahora, ¿cómo lo logra?, ¿qué tiene que ver en este escenario la innovación social?, ¿cómo las universidades pueden utilizar la innovación social para generar las competencias integrales que demanda la formación de los profesionales del siglo XXI?

\section{B. La innovación social como una estrategia académica.}

En primera instancia se requiere definir con claridad qué se entiende por innovación, puesto que es un término ampliamente utilizado en la actualidad pero que, en ocasiones, tiene múltiples intervenciones y algunas de ellas, son contradictorias o responden a apreciaciones netamente personales.

Se entiende entonces la innovación como la creación o modificación significativamente de un producto o servicio siempre y cuando éste cambio sea introducido a un mercado o rebase su alcance científico/tecnológico, en otras palabras, que responda a los interrogantes "¿qué hace?”, “por qué lo hace?”, y, “¿para quién?” o “¿para qué?”.

Ahora bien, cuando el término de innovación recibe una especificidad de tener una orientación social, se entiendo entonces como un proceso en el cual, las ideas de transformación involucran las políticas o unos servicios públicos nuevos, que brinden satisfacción a las necesidades de los ciudadanos o contribuya a la solución de las problemáticas que más están alterando el buen vivir de sus habitantes, mejorando así la eficacia de la acción del estado a cualquier nivel. 
En este sentido, generar innovación social implica también, la intervención promovida desde diferentes actores, que no siempre son lideradas por el estado o las entidades de gobierno, en ocasiones, estos procesos son orientados por organismos de la sociedad civil, e incluso, que pertenecen a la misma comunidad, porque su finalidad es que las soluciones se identifiquen con la población, se implementen con ella y a través del empoderamiento de sus habitantes, continuar en un mediano y largo plazo con la realización del mismo; de manera que, no se establezca una mal sana dependencia de los actores externos para avanzar en los procesos.

El reto más significativo está dado porque en ocasiones, estas acciones pueden suplir las funciones del estado, y con ello, es posible generar malos entendidos, en donde las entidades de gobierno tengan menores compromisos por brindar la atención oportuna y de calidad a la comunidad; además, las dificultades tienden a incrementarse cuando se confunde las gestiones de innovación social con labores asistencialistas que en últimas, contribuyen más a ahondar las condiciones de pobreza o necesidad, haciendo de ellas un negocio "rentable" para quienes lo perciben.

La innovación social no son acciones que se enmarca en pequeños periodos, que retribuyan beneficios a corto plazo, ni labores que mitiguen de forma parcial los efectos de un problema de fondo, tampoco son promesas inalcanzables que sólo aumentan las fronteras entre las organizaciones y la comunidad generando brechas que afectan el desarrollo integral de la población; todo lo contrario, con este tipo de innovación busca una contribución real a resolver problemas que afectan a una comunidad, fortaleciendo la creación de ambientes colaborativos, conectado a las personas con el objetivo de crear valor, conciencia, visibilizando el conocimiento las oportunidades y alternativas desde una perspectiva interdisciplinaria y abierta.

En conclusión, este modelo de innovación es de suma utilidad y con los diferentes metodologías de implementación, se puede de ella obtener inmensos beneficios tanto para los afectados de las problemáticas, así como también, para quienes lideran o coordinan las intervenciones; es por ello, que las universidades pueden hacer uso de este tipo de gestión para, como se manifestó anteriormente, contribuir al crecimiento de la sociedad con equidad y profesionalismo, además de generar una formación integral, humana y crítica para los jóvenes universitarios que están en su etapa educativa.

\section{Investigación Acción Participativa $-I A P-$.}

La Investigación Acción Participativa -IAP-, es una metodología de intervención comunitaria participativa que se fundamenta en el proceso investigativo para la identificación de la situación problémica, el diseño de la solución y su correspondiente implementación. Es importante enfocar la IAP como un instrumento útil para ampliar la discusión e implementación de la políticas y programas sociales, en tanto que, se plantean 
las percepciones y representaciones que tienen los sujetos involucrados acerca de sus condiciones de existencia y de desarrollo, y su interrelación con la situación, a partir de una acción directa para solucionarla, posibilitando en gran medida el éxito y la sustentabilidad de la intervención.

Para comprende cómo se implementa la IAP, es necesario hacer claridad sobre algunos conceptos, los cuales se relacionan a continuación:

- Investigación: es la acción de buscar datos para con base en ellos analizar sucesos o problemas y necesidades con un rigor científico, el objetivo de la investigación es acrecentar los conocimientos teóricos a través de su aplicación más práctica, sin interesarse directamente en sus posibles aplicaciones o consecuencias; es más formal y persigue las generalizaciones con vistas al desarrollo de teorías basadas en principios y leyes.

- Acción: desde la IAP, se entiende la acción como la asistencia, a transformar un modelo social estructural cuya finalidad es llevar a la práctica estas asistencias.

- Participación: se define como el apoyo de un grupo de personas para llevar a cabo o dar cumplimiento a un objetivo por medio de líneas de acción específicas o actividades concretas que redunden en beneficios para la comunidad.

En conclusión, el concepto IAP, se relaciona con una metodología que tiene como objetivo fundamentar el estudio de una comunidad a través de la participación de la misma, cuya finalidad es la de contribuir al desarrollo de ésta bajo las variables propias de la realidad que este grupo social experimenta en su territorio.

Actualmente, se podría entender que la IAP es un proceso metodológico que rompe los moldes de la investigación tradicional, conjuga las actividades del conocimiento de la realidad mediante mecanismos de participación con la comunidad, para el mejoramiento de su buen vivir. En su conjunto se configura como una herramienta de motivación y promoción humana, que permitiría garantizar la participación activa y democrática de la población, en el planeamiento, diseño y la ejecución de proyectos de desarrollo.

D. Contextualización de la Corporación Universitaria Autónoma de Nariño y el Programa de Inserción Rural Interdisciplinaria.

La Corporación Universitaria Autónoma de Nariño -AUNAR- es una institución de educación superior comprometida con la cultura, la ciencia, la investigación; la excelencia en la formación de profesionales íntegros y el liderazgo en el desarrollo social; esta organización tiene presencia en el territorio colombiano a través de su sede principal, ubicada en la ciudad de Pasto, y cinco extensiones más que se encuentran en Ipiales (Departamento de Nariño), Puerto Asís (Departamento de Putumayo), Cali (Departamento 
del Valle del Cauca), Villavicencio (Departamento de Meta) y Cartagena (Departamento de Bolívar). Actualmente, tiene una población estudiantil de alrededor de 6.000 estudiantes.

En Chile durante el año de 1990 surge una epidemia de hepatitis viral, bajo este contexto el departamento de salud pública de la Universidad de la Frontera, asume el reto social de contribuir a la construcción de una solución hacia esta problemática, desarrollando actividades como: Programas de prevención de la enfermedad, investigación, apoyo y reforzamiento de los centros de atención de salud y la generación de redes de relaciones colaborativas con las diversas instituciones y organizaciones locales, en razón de ello, en el año de 1991 realizan el Programa de Internado Rural Interdisciplinario -PIRI-, con un objetivo es vincular a los estudiantes con el entorno, con el fin de descubrir el gran potencial de un territorio como escenario de aprendizaje y formación profesional. (UNIVERSIDAD DE LA FRONTERA., 2013).

El municipio de Pasto capital del departamento de Nariño cuenta con una población total de 439.993 habitantes (el 25,2 por ciento de la población total del Departamento de Nariño); de los cuales, el 51,7 por ciento son hombres (227.611) y el 48,3 por ciento son mujeres; en cuanto a su distribución espacial, el 83,1 por ciento (365.651 habitantes), se ubica en la parte urbana del municipio y el porcentaje restante $(16,9 \%)$ en el sector rural. (Secretaría de Salud Municipal, 2016).

La Corporación Escuela del Sur es una organización sin ánimo de lucro que tiene como misión asesorar, implementar, planear, gestionar y ejecutar actividades encaminadas a la búsqueda de la protección del medio ambiente, desarrollo sostenible, la resolución de conflicto socio-ambientales, el desarrollo social y cultural de las comunidades, así como la contribución a la construcción de la paz con un cierto grado de incidencia política.

Bajo ese contexto con la coordinación de su directora, Angélica Chavarría Lagos, se desarrolla el Programa de Inserción Rural Interdisciplinario -PIRI-, mediante un proyecto presentado a la Vicerrectoría de Investigaciones, Postgrado y Relaciones Internacionales VIPRI-, comenzando con la ejecución de las acciones en el año 2014, momento en el cual se participó con estudiantes de la Universidad de Nariño con quienes se formó un equipo interdisciplinario integrado por profesionales de las diferentes facultades tales como: ingeniería agroforestal, agronomía, sociología, psicología, promoción de salud y administración de empresas.

En el año 2015 durante el periodo B, mediante el convenio de cooperación interinstitucional firmado por la AUNAR con Corporación Escuela del Sur, se permitió que los universitarios de esta Institución de Educación Superior, fueran parte del equipo PIRI en el Municipio de Pasto; incorporando inicialmente estudiantes de administración de empresa e ingeniería mecánica, quienes trabajaron conjuntamente con otros jóvenes universitarios de ingeniería ambiental, sociología y administración de empresas de la Universidad de Nariño; 
este equipo comenzó un nuevo procesos en el corregimiento El Encano- Municipio de Pasto; durante este periodo se formaron las siguientes líneas de acción:

- Emprendimiento sostenible cuyo objetivo fue motivar a los estudiantes del colegio en torno a la oportunidad de emprender con enfoque de sostenibilidad.

- Manejo adecuado de residuos sólidos su objetivo fue crear herramientas de sensibilización comunitaria respecto del adecuado manejo de residuos sólidos.

- Exploratoria de bioarquitectura objetivo fue indagar la disposición comunitaria a la creación de hitos turísticos bioarquitectónicos.

- Ecoturismo como una oportunidad para el desarrollo local su objetivo fortalecer estrategias de turismo sostenible en coordinación con organizaciones y comunidad.

- Fortalecimiento cultural su objetivo realizar un ejercicio de Memoria histórica del territorio y transformarlo en icono local y turístico.

- Monitoreo y evaluación del plan de adaptación al cambio climático su objetivo fue Identificar un esquema participativo de monitoreo y evaluación del Plan de Adaptación al Cambio Climático y su sistema de alertas tempranas.

\section{Articulación de la innovación social y la proyección social. Una mirada al entorno y una contribución hacia él}

A partir de la experiencia vivida con el Programa de Internado Rural Interdisciplinario y por los resultados obtenidos con él, se ha establecido un modelo de gestión que articule la innovación social con la proyección de la universidad hacia la comunidad, cuya metodología se describe a continuación, así como también los resultados en él esperados, el esquema de trabajo desde la gestión universitaria y los beneficios en el proceso de formación profesional brindado a los estudiantes universitarios.

Desde lo metodológico la innovación social debe contar con la formación de trabajo con la comunidad, centrándose en el interés de los estudiantes a través del desarrollo integral desde la singularidad de los procesos de enseñanza aprendizaje y la inserción intervención.

El fundamento metodológico se interrelacionan tres niveles de integración a la innovación social: la integración del estudiante y la docencia, la investigación aplicada y la acción social, con estos tres niveles se pretende saber, hacer, ser, conocer, interpretar, actuar, pensar, vivir, convivir, liderar y emprender con el objetivo de realizar propuestas conjuntas de acción social, para la resolución de problemas, capaces de crear mejores condiciones de vida en aquellos grupos sociales carente de ellas. 
Bajo este contexto, el estudiante desarrolla conocimientos teóricos-prácticos del trabajo social, que le sirven también a la universidad para proyectarse hacia la comunidad, en donde se fortalecen habilidades y destrezas para el conocimiento e intervención de:

- Organizaciones populares

- Desarrollo de espacios de intervención del Trabajo Social en la comunidad.

- Diagnóstico participativo con la comunidad.

- Investigación participativa e interdisciplinaria.

- Proceso de inserción e intervención con las comunidades.

Estos conocimientos fortalecen las habilidades, actitudes, aptitudes y perspectivas de las competencias, mediante el aprendizaje que ayuda abordar las problemáticas sociales y el manejo adecuado tanto de las herramientas como de los métodos para operar eficazmente; fortaleciendo competencias de efectividad personal o ciudadanas, este último término de acuerdo a los lineamientos establecidos por el Ministerio de Educación Nacional. En el siguiente cuadro se resume las competencias a fortalecer y sus correspondientes indicadores de evaluación.

\section{COMPETENCIA}

Incrementar el conocimiento por

parte de los estudiantes sobre las necesidades de la comunidad.

Formar estudiante íntegros que conduzcan a obrar, sentir, y respetar en todo momento con valores morales, las buenas costumbres y prácticas profesionales, respetando las políticas organizacionales de la comunidad.

Potencializar el aprendizaje
continuo y la capacidad integral de
$\begin{array}{lll}\text { ampliar los } & \text { conocimientos } \\ \text { profesionales } & \text { utilizando las } \\ \text { relaciones } & \text { interdisciplinaridad }\end{array}$

\section{INDICADOR CUALITATIVO}

Entender las necesidades de la comunidad.

Tener perspectiva de los puntos de vista de otras personas.

Crear una buena relación y credibilidad con los profesionales colaboradores y comunidad.

Demostrar confiabilidad y profesionalismo al tratar con los compañeros, comunidad y miembros del equipo.

Responder de manera coherente a situaciones que requieren honestidad y franqueza.

Evitar conflictos entre el trabajo y los intereses o actividades personales.

Demostrar interés en el aprendizaje y desarrollo personal.

Modificar el comportamiento basándose en los comentarios o el auto-análisis de equivocaciones. 
Articulación de la proyección social con la gestión universitaria en innovación social. Una mirada hacia la realidad del entorno y la contribución hacia él

$\begin{aligned} & \text { como una oportunidad para } \\ & \text { fortalecer la formación tanto } \\ & \text { técnica como personal. }\end{aligned}$
Aumentar la comunicación
efectiva focalizando al estudiante
en crear y mantener contactos
amistosos con personas que son o
serán útiles para alcanzar las metas
relacionadas con el trabajo
comunitario.

Fortalecer las habilidades

Interpersonales que implica la convicción necesaria para mejorar, innovar, motivar y promover el espíritu emprendedor entre sus colaboradores y cuyo objetivo es la cooperación entre ellos para lograr los objetivos planteados.

Aumentar la creatividad
profundizándose en la capacidad
del estudiante en modificar las
cosas, partiendo de formas o
situaciones no pensadas, tiene como
finalidad crear soluciones nuevas y
diferentes ante problemas o
situaciones requeridos en el trabajo
con comunidades.

Buscar entrenamiento o formación complementaria que apoye los objetivos de la carrera profesional.

Hablar con claridad y confianza; organizar la información de una manera lógica, hablar usando lenguaje convencional, incluyendo una gramática, tono y ritmo apropiados.

Practicar la comunicación significativa bidireccional, hablando con claridad; prestando especial atención y buscando entender a los demás, escuchar atentamente, aclarar la información y prestar atención a las señales no verbales $\mathrm{y}$ responder adecuadamente.

Influenciar a los demás; presentar los pensamientos e ideas de forma persuasiva, lograr el compromiso y asegurar el apoyo a las ideas propuestas.

Relacionarse bien con la comunidad, colegas profesionales y miembros del equipo.

Crear un entorno abierto que anime a las personas a trabajar conjuntamente para resolver problemas y mejorar prácticas y servicios.

Analizar y resolver los conflictos a medida que estos surgen.

Demostrar una curiosidad intelectual acerca del por qué las cosas.

Demostrar una tendencia hacia la acción; materializar ideas en productos o servicios.

Tabla 1. Competencias desarrolladas por el estudiante en un proyecto con el enfoque en innovación social. 
Estas competencias se alcanzan cuando desde la gestión universitaria en proyección social articule la innovación social a través de un esquema de trabajo que se podría sintetizar de la siguiente manera:

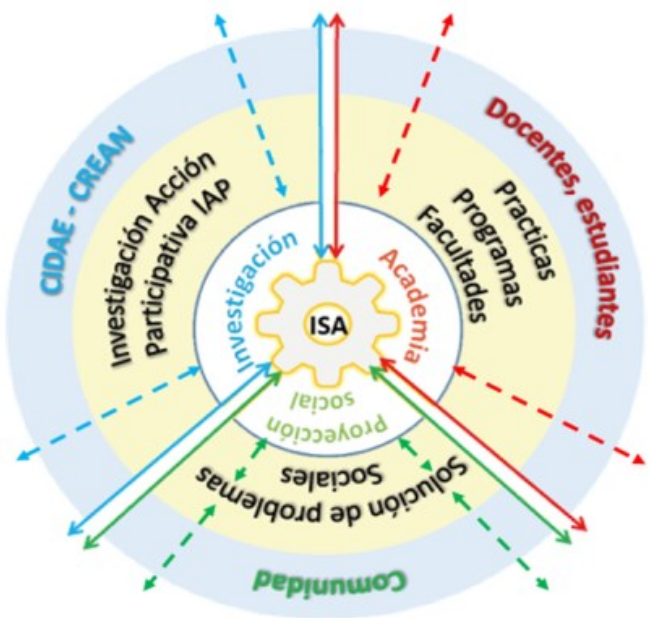

Figura 1. Gestión Universitaria en innovación social.

Bajo este modelo se genera una articulación del emprendimiento universitario, las labores investigativas, las acciones académicas curriculares como lo son las prácticas profesionales y el desarrollo de algunas asignaturas, en conjunto con la comunidad, con quienes no solo se trabaja sinérgicamente, sino se vincula al proceso de formación y acción universitaria.

Como resultado, se busca poner a prueba las habilidades del participante, a través de la aplicación de los conocimientos previamente adquiridos, cuyo propósito es entender las necesidades de la comunidad, comprender la perspectiva de otras personas, para crear una buena relación entre los profesionales jóvenes, los colaboradores, la universidad y la comunidad.

Bajo estos procesos, se cumple con el objetivo de mejorar el desarrollo de la innovación social, mediante la interrelación del conocimiento, la interdisciplinariedad y la investigación con impacto social, fomentando la cooperación dentro de la universidad, además de fortalecer las relaciones con otras instituciones que apoyan la innovación social dentro de la región. 
Articulación de la proyección social con la gestión universitaria en innovación social. Una mirada hacia la realidad del entorno y la contribución hacia él

\section{Conclusión}

El proyecto de investigación aporta a la academia un modelo de gestión universitaria en innovación social que funciona como un mecanismo concreto de relacionamiento y cooperación con el territorio, además de facilitar una interacción directa de los estudiantes con la sociedad permitiéndoles enfocar sus conocimientos adquiridos de forma más práctica interdisciplinar y transversal.

Este modelo de gestión otorga herramientas que contribuyen en el fortalecimiento de una cultura innovadora de alto impacto, potencializando las competencias de los estudiantes, además de aportar en el cumplimiento de los procesos misionales y los retos que afronta la educación superior en la actualidad.

\section{Referencias}

Cossette, P. (Abril, 2011). Diez reglas de la publicación en una revista académica. Ediciones Uniandes. Universidad de los Andes. Bogotá-Colombia.

Mesa, J. \& Osorio, D. (noviembre, 2016). Innovación a la M. Universidad EAFIT. Medellín, Colombia.

Morales, A. (2008). Innovación social: una realidad emergente en los procesos de desarrollo. Revista de Fomento Social 63. Universidad Loyola Andalucía. Córdoba-España, 411-444.

Newell, S. (2002). Creando organizaciones saludables. Bienestar, diversidad y ética en el trabajo. Thomson Learning. Madrid, España.

Pérez, S.\& Castaño, R. (2016). Funciones de la Universidad en el siglo XXI: humanística, básica e integral. Revista Electrónica Interuniversitaria de Formación del Profesorado, 19(1), 191--- 199.

Rodríguez, A. \& Alvarado, H. (noviembre, 2008). Claves de la innovación social en América latina y el Caribe. Comisión Económica para América Latina y el Caribe (CEPAL). Santiago de Chile.

Secretaría de Salud Municipal. (2016). Plan Territorial de Salud. Pasto: Alcaldía de Pasto.

UNIVERSIDAD DE LA FRONTERA. (2013). Programa de Internado Rural Interdisciplinario (P.I.R.I). . Obtenido de http://piri.ufro.cl/index.php/piri/historia> 